\title{
Radar backscatter and forest biomass
}

To the Editor - In relation to the use of radar backscatter to estimate forest biomass, Woodhouse et al. ${ }^{1}$ state that our study ${ }^{2}$ provides an extreme example of a common analytical error, namely fitting a log function instead of a sigmoidal function. In fact, there is almost no difference between these functions, because the slope of the $\log$ function is very small in higher biomass ranges.

Furthermore, they criticize us for using the fitted function and not the data to calculate the saturation. The authors make the incorrect statement that the fitted function is used to project sensitivity to aboveground biomass (AGB) values higher than 600 tonnes per hectare $\left(\mathrm{t} \mathrm{ha}^{-1}\right)$. Most studies on radar and AGB estimate the saturation through visual interpretation. In contrast, we calculated the saturation level on the basis of the radiometric accuracy of the radar data and a chosen accuracy level. We used two accuracy levels (50 and $100 \mathrm{t} \mathrm{ha}^{-1}$ ) and clearly state in the paper that "within the accuracy interval of $50 \mathrm{t} \mathrm{ha}^{-1}$ the estimations are supposed to be accurate whereas estimations within the $100 \mathrm{tha}^{-1}$ accuracy interval are only indicators for the spatial AGB distribution." We found a maximum saturation of $300 \mathrm{tha}^{-1}$ at the accuracy level of $50 \mathrm{t} \mathrm{ha}^{-1}$ and emphasized that the $100 \mathrm{t} \mathrm{ha}^{-1}$ saturation level is not accurate enough for a reliable AGB estimation.

Woodhouse et al. contest the wording 'direct measurement'. However, we stated prominently that "no remote sensing can directly measure biomass". If the whole abstract is read, it is clear that we speak of AGB estimations based on radar, even if we use the term 'direct measurement'.

By using the term 'direct AGB estimation/measurement', we refer to the 'direct remote sensing approach' to estimate AGB, introduced by Goetz et al. ${ }^{3}$, for which radiometric satellite measurements are calibrated to field-based AGB values. In contrast, the 'indirect AGB estimation' refers to the 'stratify and multiply approach' which links a biomass value determined for a specific vegetation type to a remote-sensing-based land-cover map.

In our opinion, it makes sense to emphasize the difference between these two completely different biomass estimation approaches as they lead to differing results, which we also showed in our paper ${ }^{2}$.

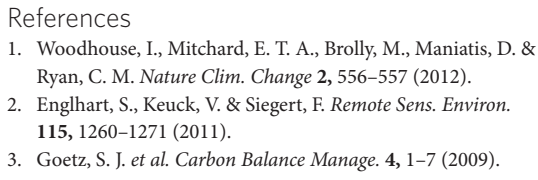

S. Englhart ${ }^{1 \star}$, V. Keuck ${ }^{2}$ and F. Siegert ${ }^{1,2}$

'Biology Department II, GeoBio Center, LudwigMaximilians-University, Großhadener Straße 2, D-82152 Planegg-Martinsried, Germany,

${ }^{2}$ Remote Sensing Solutions $\mathrm{GmbH}$, Isarstraße 3, D-82065 Baierbrunn, Germany.

*e-mail: englhart@rssgmbh.de

\section{Forest biomass and the science of inventory from space}

To the Editor - Although we agree that the term 'direct measurement' of aboveground biomass in the remotesensing literature is inappropriate, Woodhouse et al. ${ }^{1}$ wrongly criticize some valid results in the literature and paint a misleading picture of the current state of the art in radar remote sensing.

Aboveground biomass (AGB) can only be measured directly by destructively harvesting trees, and measuring the mass by scales calibrated to an internationally acceptable standard ${ }^{2}$. In all field inventory and remote-sensing techniques, measurements of forest attributes are used in a model to estimate biomass and report errors ${ }^{3}$. However, the studies cited by Woodhouse et al. ${ }^{1}$ explicitly identify their methods as indirect estimations and provide associated errors, using the term to distinguish estimates based directly on backscatter from those that first estimate height and then use this to infer biomass.

Quantifying uncertainty is challenging for both field inventory and remotesensing estimations $s^{3,4}$. Uncertainty is quantified in terms of accuracy and precision, with accuracy being the difference between an estimate and the true value, and precision being the reproducibility or the variance among repeated estimates ${ }^{2}$. Thus, good accuracy is always more difficult to obtain than good precision. A precise estimate of forest biomass can usually be achieved with both replicated remote-sensing or field inventory estimates. For precise radar estimates of $\mathrm{AGB}$, remote-sensing estimates must be corrected for factors such as changing soil moisture or topography ${ }^{5}$. However, field-inventory methods also suffer from methodological biases $^{3}$. With careful selection of ground data for calibration of radar models, relative error of about $20 \%$ can be achieved on biomass for forest stands with mixed species and complex landscapes ${ }^{5}$. Hence, radar or lidar remote-sensing techniques from space can provide systematic and accurate estimates of AGB. Once calibrated with limited but unbiased forest inventory samples, such estimates not only represent an alternative to conventional field inventory methods but, unlike field inventory data, allow spatially refined 